\title{
Homogeneous nucleation in associated vapors. II. Formic and propanoic acids
}

\author{
Yvonne G. Russell \\ Richard H. Heist \\ Fairfield University, rheist@fairfield.edu
}

Follow this and additional works at: https://digitalcommons.fairfield.edu/engineering-facultypubs

(c) 1978 American Institute of Physics.

The final publisher PDF has been archived here with permission from the copyright holder. https://aip.scitation.org/doi/abs/10.1063/1.437035

\section{Peer Reviewed}

\section{Repository Citation}

Russell, Yvonne G. and Heist, Richard H., "Homogeneous nucleation in associated vapors. II. Formic and propanoic acids" (1978). Engineering Faculty Publications. 155.

https://digitalcommons.fairfield.edu/engineering-facultypubs/155

\section{Published Citation}

Russell, Y. G., \& Heist, R. H. (1978). Homogeneous nucleation in associated vapors. II. Formic and propanoic acids. The Journal of Chemical Physics, 69(8), 3723-3728. doi:10.1063/1.437035.

This item has been accepted for inclusion in DigitalCommons@Fairfield by an authorized administrator of DigitalCommons@Fairfield. It is brought to you by DigitalCommons@Fairfield with permission from the rightsholder(s) and is protected by copyright and/or related rights. You are free to use this item in any way that is permitted by the copyright and related rights legislation that applies to your use. For other uses, you need to obtain permission from the rights-holder(s) directly, unless additional rights are indicated by a Creative Commons license in the record and/or on the work itself. For more information, please contact digitalcommons@fairfield.edu. 


\title{
Homogeneous nucleation in associated vapors. II. Formic and propanoic acids
}

\author{
Yvonne G. Russell and Richard H. Heist ${ }^{\text {a) }}$ \\ Department of Chemical Engineering, University of Rochester, Rochester, New York 14627 \\ (Received 23 May 1978) \\ Homogeneous nucleation measurements have been made on formic and propanoic acids. The temperature \\ dependence of the critical supersaturation was measured and found to agree well with that predicted by \\ the Katz-Saltsburg-Reiss theory for nucleation in associated vapors.
}

\section{INTRODUCTION}

For more than 50 years since the pioneering work of Volmer and Weber, the theory of nucleation has been tested and refined to a point where there is little doubt that for relatively simple, nonreacting chemical systems it does a rather remarkable job in predicting the onset of observable nucleation.

The situation with regard to more complex chemically reacting systems is much less certain. The existing literature is scarce and deals almost entirely with the theory of vapor to liquid homogeneous nucleation in associated vapors. Even this, however, has done little to answer the crucial questions about the effect of association upon nucleation rate since several authors ${ }^{1}$ predict a decrease in nucleation rate with an increasing degree of association while others ${ }^{2}$ predict an increase in nucleation rate.

There appear to have been only two attempts to actually observe homogeneous nucleation in vapors exhibiting large degrees of association. The first, over 70 years ago, was a series of cloud point measurements made in a Wilson cloud chamber involving several low molecular weight carboxylic acids and their corresponding esters. ${ }^{3}$ These data are suspect, however, because of questions regarding the vapor temperature after the expansion. ${ }^{4}$ The second and most recent set of experiments was a careful measurement of the variation of critical supersaturation with temperature of acetic acid vapor in an upward thermal diffusion cloud chamber. ${ }^{5}$ These latter experiments clearly indicate a dramatic decrease in the nucleation rate of acetic acid vapor at temperatures where the vapor is undergoing extensive association. In fact, in this paper (referred to hereafter as I), the authors point out that, with respect to the nucleation rate in a hypothetical vapor of pure acetic acid monomer, the reduction in nucleation rate upon passing to a vapor which is roughly 0.91 mole fraction dimer (characteristic of their experiments) is of order $10^{18}$.

This paper reports results of new homogeneous nucleation measurements of highly associated vapors which extend the experiments reported in I to formic acid and propanoic acid.

\section{COMMENTS ON EXPERIMENT DESIGN AND OPERATION}

The diffusion cloud chamber used for the experiments described in this paper is similar both in design and

a) To whom all correspondence should be addressed. operation to the cloud chamber denoted as CC2 described earlier in I. There are, however, a few important differences. First of all, only the lower plate in this study was Teflon coated. The upper plate surface was uncoated aluminum. The reason for this was simply to make use of the fact that both formic and propanoic acids wet the aluminum surface and form smooth, uniform films of condensate. During all our experiments, the temperature of the upper plate never exceeded $290 \mathrm{~K}$ so that chemical attack of the aluminum by either acid was not a problem. In fact, the upper plate surface never lost the original mirror finish. Operating without a Teflon coated upper plate obviates using a thin layer of glass wool to promote wetting and proper drainage of the condensate film. ${ }^{6}$

There were no inlet ports in the upper plate as shown in Fig. 1 of I. Rather, a $0.95 \mathrm{~cm} \mathrm{o.d.} \mathrm{Pyrex} \mathrm{tube} \mathrm{was}$ fused to the Pyrex ring separating the two chamber plates, and this provided access to the chamber interior. Viton gaskets were used to provide a seal between the Pyrex ring and the upper and lower chamber plates. Although Viton is generally recommended for use with these materials, it could not withstand prolonged exposure to either acid. Usually, after an hour or so, the working fluid pool would noticeably discolor, and the gaskets would begin to swell, leading eventually to fracture. The only way to extend gasket life was to empty the chamber after several experiments, rinse the interior thoroughly with distilled water (keeping it completely assembled), and bake and evacuate the chamber for 10 to $12 \mathrm{~h}$. This process could be repeated two or three times for propanoic acid but was not effective for formic acid. We were never able to complete more than one experiment at a time while using formic acid. We attempted to use teflon gaskets but found them to be too hard to provide a vacuum seal between the gasket and the teflon coated surface. We were not able to compress these gaskets sufficiently for fear of fracturing the Pyrex ring.

The temperatures of both plates were controlled by constant temperature circulation baths. The upper and lower liquid surface temperatures were measured directly with flat, $0.01 \mathrm{~cm}$ thick chromel-alumel thermocouples brought into the chamber through the Pyrex ring in a fashion described in detail in I. The temperature stability of the upper plate liquid film was good, never varying more than $0.1 \mathrm{~K}$ during any one experiment. The lower plate pool was considerably less stable. This was due in part to the depth of the pool $(2-4 \mathrm{~mm})$ and to the rather high temperatures necessary to maintain a 


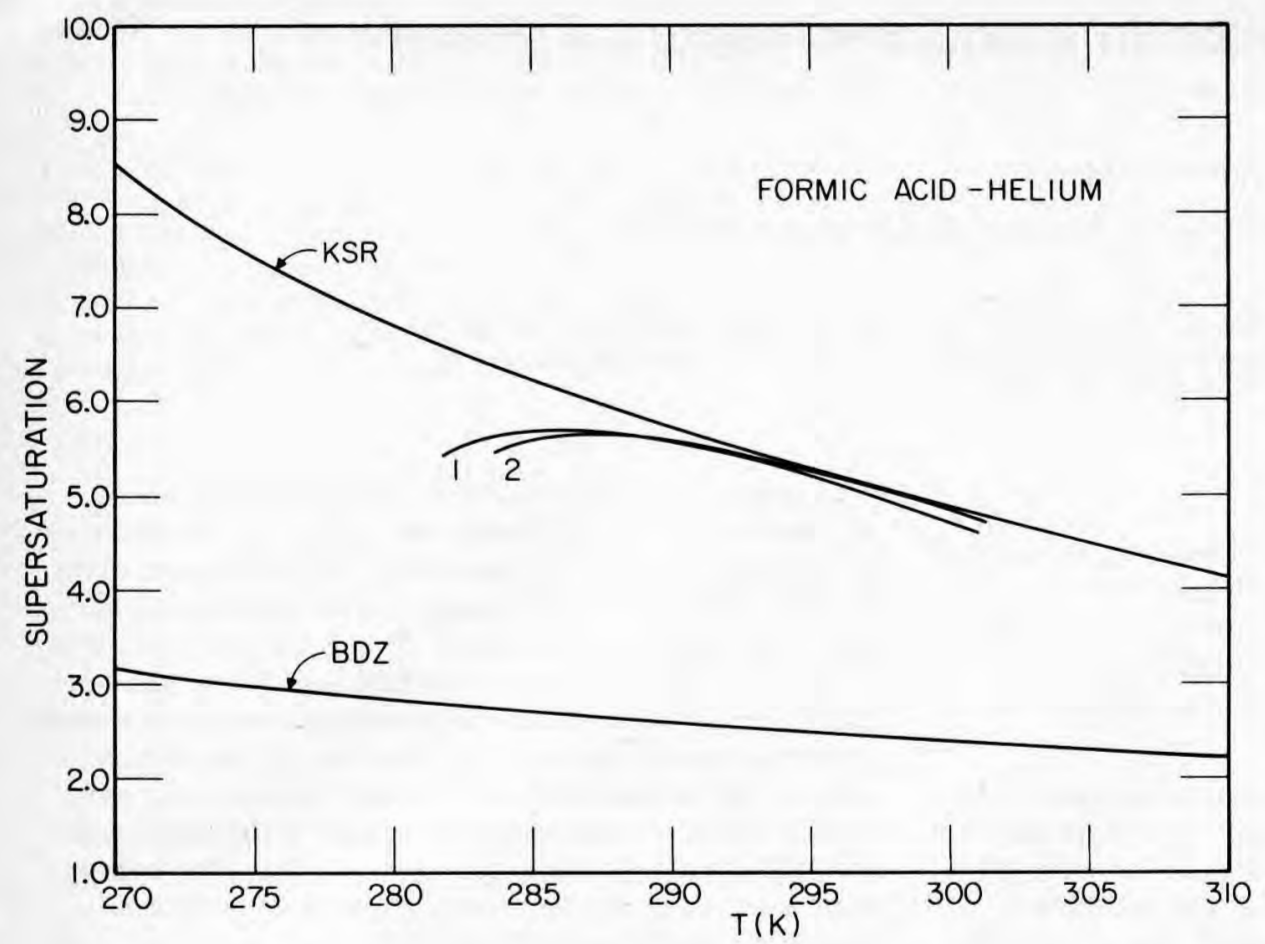

FIG. 1. The variation of the critical supersaturation of formic acid vapor as a function of temperature. The curves labeled BDZ and KSR represent the predictions of the Becker-Döring-Zeldovitch theory and the Katz-Saltsburg-Reiss theory, respectively. The envelope of the numbered curves is the experimentally measured variation.

finite nucleation rate. Periodic variations in the surface temperature of $0.5 \mathrm{~K}$ were not uncommon.

Nucleation was observed by directing a collimated, heat filtered light beam from a $600 \mathrm{~W}$ tungsten iodide lamp through the chamber and observing the forward scattered light from the falling droplets. It was easy to discern when conditions were no longer stable inside the chamber. If there was gasket fracture or thermocouple feed-through failure, we would observe either falling drops with curved trajectories and/or nonuniform nucleation rates throughout the chamber.

The basic principles of diffusion cloud chamber operation and a typical procedure for any one experiment have been discussed elsewher $e^{5-7}$ and need not be reviewed here.

Both carboxylic acids used in this study were obtained from Eastman Organic Chemicals. The original "as is" formic acid was guaranteed to only $95 \%$ purity, the reason being that formic acid undergoes a continual thermal decomposition to water and carbon monoxide. ${ }^{8}$ We analyzed this formic acid and found it to be $97.6 \%$ pure. Using standard techniques, ${ }^{8}$ we purified the original stock material until our samples were at least $99.2 \%$ formic acid. We shall return to this point later. The propanoic acid was assayed at $99 \%$ purity. Airco 99. 995\% Helium gas was used as a carrier gas for all our experiments. No attempt was made to purify further the propanoic acid or the Helium carrier gas. Thermocouple voltages were measured with a Leeds and Northrop $\mathrm{K}-4$ potentiometer in conjunction with a Keithly model 155 microvolt null detector. Trends in the Thermocouple voltages were followed using a Houston omniscribe chart recorder.

\section{TEMPERATURE AND PRESSURE PROFILES}

In order to determine the vapor supersaturation inside the diffusion cloud chamber, it is necessary to solve the coupled energy and mass transport equations which describe the energy and mass fluxes between the two chamber plates. Careful choice of chamber geometry simplifies this problem somewhat since, at large aspect (diameter: height) ratios, the transport processes can be approximated as unidimensional; but the problem is still sufficiently complicated that even for simple two component systems, e.g. , water and helium, the equations do not admit to a convenient analytical solution. Rather, they must be solved numerically to yield the temperature and pressure profiles in the chamber and ultimately the vapor supersaturation. This problem becomes considerably more complex when there are more than two components in the chamber and in the presence of a chemical reaction.

This is the problem we face in making homogeneous nucleation measurements on formic and propanoic acid vapors. It is well known that both these substances exhibit large degrees of association in the vapor, and that the vapor composition is essentially that characterized by the equilibrium association reaction ${ }^{9-11}$

$$
2 \mathrm{RCOOH} \rightleftharpoons(\mathrm{RCOOH})_{2} \text {, }
$$

where $\mathbf{R}=\mathbf{H}$ or $\mathrm{CH}_{3} \mathrm{CH}_{2}$ for formic or propanoic acid, respectively. With this model for the vapor composition, approximate expressions which describe the mass and energy transport in acetic acid vapor have already been developed in I. We shall use this same formalism here with only slight modification.

Neglecting all effects of thermal diffusion, all contributions to diffusion arising from external forces and 
gradients, assuming a perfect gas mixture of acid monomer, acid dimer, and carrier gas, and assuming local equilibrium throughout the chamber, it has been shown ${ }^{5}$ that (for the steady state, plane parallel diffusion conditions which prevail in the chamber) the pressure and temperature profiles can be written as

$$
\frac{d P}{d Z}=\frac{\left(P-P_{T}\right) \beta}{C D_{13}} L
$$

and

$$
d T / d Z=\lambda^{-1}(L H-Q),
$$

respectively. In Eq. (2), $P=P_{1}+P_{2}$ is the total partial pressure of the diffusing species (monomer plus dimer), $P_{T}$ is the total chamber pressure, $Z$ is the reduced chamber height $z / h$, where $h$ is the actual chamber height, $C$ is the total vapor concentration, and $D_{13}$ is the monomer-carrier gas binary diffusion coefficient. $L$ is the reduced molar flux given by $(l h) / M_{1}$, where $l=l_{1}$ $+l_{2}$ is the total mass flux ( $z$ direction) and $M_{1}$ is the molecular weight of the monomer, and $\beta=1$ $-0.29 \phi$. Here, $\phi=l_{2} / l$ is the dimer flux fraction. In Eq. (3), $\lambda$ is the mixture thermal conductivity, and $Q$ is the reduced energy flux $q h$, with $q$ being the energy flux and $H=H_{1}+\phi\left(H_{2} / 2+H_{1}\right)$, where $H_{1}$ and $H_{2}$ are the monomer and dimer molar enthalpys, respectively.

The solution of Eqs. (2) and (3) which give the pressure and temperature profiles in the chamber has previously been discussed in I. The values for the various thermodynamic and hydrodynamic properties used in their solution are summarized in Table I. An increasingly important problem with cloud chamber experiments on the more complex chemical systems is the lack of necessary data needed to evaluate the transport equations. ${ }^{5,12}$ For instance, data on the vapor thermal conductivity of formic and propanoic acids is almost nonexistent. It is $\mathrm{known}^{13,14}$ that, for reacting vapor mixtures, the thermal conductivity can be expressed as $\lambda=\lambda_{f}+\lambda_{R}$, where $\lambda_{R}$, the contribution to the total ther mal conductivity due to the chemical reaction, can be much larger than the "frozen" thermal conductivity $\lambda_{f}$, which ignores the reaction. Butler and Brokaw ${ }^{15,16}$ give as an expression for $\lambda_{R}$ as

$$
\lambda_{R}=\frac{D_{12} P}{R T}\left(\frac{\Delta H^{2}}{R T^{2}}\right) \frac{x_{1} x_{2}}{\left(n x_{2}+x_{1}\right)^{2}},
$$

where, in our case, $D_{12}$ is the binary diffusion coefficient for the monomer-dimer mixture, $\Delta H$ is the enthalpy change accompanying the association-dissociation reaction in Eq. (1), $x_{1}$ and $x_{2}$ are, respectively, monomer and dimer mole fractions, and $n$ the stoichiometric coefficient in Eq. (1), Using Eq. (4) along with the method outlined in Renner et al. ${ }^{17}$ to estimate $D_{12} P$ and the modified Eucken factor method to calculate $\lambda_{f}$, we obtain the expressions for the vapor thermal conductivity for formic and propanoic acids given in Table I. While the calculated values for propanoic acid are in fairly good agreement with available data from the literature, ${ }^{14}$ no experimental data for formic acid vapor could be lound. Experimental data for the vapor viscosity of either acid were not available in the temperature range of interest and had to be estimated. ${ }^{18}$

While some data for the heat capacity of formic acid monomer were available, ${ }^{19}$ values for the dimer were not. In addition, heat capacity data for both propanoic acid monomer and dimer could not be found. The group contribution method of Rihani and Doraiswamy ${ }^{20}$ was used to estimate both the monomer and dimer heat capacities. This method works reasonably well for the monomer, but is only a rough approximation for the dimer. For instance, it gives dimer heat capacities that differ by slightly less than $10 \%$ from calculated values for acetic acid ${ }^{21}$ and by less that $5 \%$ for the $\mathrm{NO}_{2}-\mathrm{N}_{2} \mathrm{O}_{4}$ system. ${ }^{22}$ Fortunately, the resulting supersaturation profiles are not very sensitive to the precise value of the heat capacity. We shall return to this point later.

The monomer-carrier gas binary diffusion coefficients $D_{13}$ in Eq. (2) were estimated using the method of Fuller, Schettler, and Giddings. ${ }^{23}$

\section{RESULTS AND DISCUSSION}

In Table II, we list the experimental values for the upper plate condensate film temperature $T_{1}$, the surface temperature of the working fluid pool on the lower plate $T_{0}$, and the total pressure $P_{T}$ in the chamber. Each set of these conditions corresponds to an experiment in which the nucleation rate was uniform throughout the chamber and constant at a value of $1-3 \mathrm{drops} /\left(\mathrm{cm}^{3} \mathrm{~s}\right)$. This data is used in solving Eqs. (2) and (3) for the pressure and temperature profiles in the chamber and ultimately the variation in total supersaturation. Here, total supersaturation refers to both monomer and dimer contributions. This supersaturation profile is then plotted versus temperature for each experiment. These plots (the numbered curves) are shown in Fig. 1 for formic acid and in Fig. 2 for propanoic acid. Just the portion of the profile with the largest values of the supersaturation has been plotted. The envelope of these curves is the experimentally determined variation of critical supersaturation with temperature. In Figs. 1 and 2, the actual envelopes have not been drawn simply for the sake of clarity.

The solid curve in each plot labeled $\mathrm{BDZ}$ is the variation of critical supersaturation with temperature of a hypothetical vapor of acid monomer as given by the Becker-Döring-Zeldovitch theory of homogeneous nucleation. The rate of nucleation in this theory is given by

$$
J^{\prime}=\frac{a}{d}\left(\frac{2 N_{0}^{3} \sigma M}{\pi}\right)^{1 / 2}\left(\frac{S P_{e}}{R T}\right)^{2} \exp \left[-\frac{16 \pi N_{0}}{3(\ln S)^{2}}\left(\frac{M}{d}\right)^{2}\left(\frac{\sigma}{R T}\right)^{3}\right],
$$

where $J^{\prime}$ is the nucleation rate in drops $/\left(\mathrm{cm}^{3} \mathrm{~s}\right) ; a$ is the accommodation coefficient, $d$ the bulk liquid density, $\sigma$ the bulk surface tension, $P_{e}$ the equilibrium vapor pres sure, $M$ the molecular weight, $N_{0}$ the Avogadro number, and $R$ the gas constant. $S$ in Eq. (5) is the supersaturation ratio defined as $P / P_{e}$ where $P$ is the existing pressure of the vapor. The BDZ curves in Figs. 1 and 2 are calculated setting $J^{\prime}=1$ in Eq. (5) and solving for $S$ at each temperature. The accommodation coefficient was assumed to be unity in all cases.

In both Figs. 1 and 2 , the actual variation of the critical supersaturation with temperature (the envelope of the numbered curves) is clearly higher than the $\mathrm{BDZ}$ prediction (the nucleation rate is slower). In fact, the measured nucleation rate in our experiments at $295 \mathrm{~K}$ 
TABLE I. Expressions for the vapor thermal conductivity $\lambda$, saturation vapor pressure $P_{e}$, surface tension $\sigma$, vapor viscosity $\eta$, liquid density $d$, heat capacities for the monomer $C_{p_{1}}$ and dimer $C_{p_{2}}$, and association equilibrium constant $K_{12}$ for each acid and those for the carrier gas thermal conductivity $\lambda$ and viscosity $\eta$. Values for the molecular weight $M$ the binary diffusion coefficients $D_{13}$ and the binary diffusion coefficient temperature dependence $S .^{2}$

\section{Formic acid}

$$
\begin{aligned}
\lambda= & \frac{\left[2.82 \times 10^{-7}+4.16 \times 10^{-7}\left(C_{p_{1}}\right)\right] T^{4.5}}{(T+466)} \\
& +\frac{27.03+2.56 \times 10^{-3} T-3.39 \times 10^{-6} T^{2}}{T^{0.5}(T+466)}{\frac{x_{1} x_{2}}{\left(2 x_{2}+x_{1}\right)^{2}}}^{b}
\end{aligned}
$$

$\log P_{e}=7.858-\frac{1860}{T}^{c}$

$$
\sigma=69.244-0.108 T^{d}
$$$$
\eta=145.1 \times 10^{-7} \frac{T^{1.5}}{(T+466)}
$$$$
d=1.5779-1.2207 \times 10^{-3} T^{8}
$$$$
C_{p 1}=4.168+2.507 \times 10^{-2} T-8.222 \times 10^{-6} T^{2 \mathrm{~B}}
$$$$
C_{\rho_{2}}=8.336+5.014 \times 10^{-2} T-1.644 \times 10^{-5} T^{2 \mathrm{~h}}
$$

$\log K_{12}=\frac{3037}{T}-10.616^{1}$

$$
\begin{aligned}
M & =46.03 \\
D_{13} & =0.4587 \frac{T}{C}^{j} \\
s & =0.75^{j}
\end{aligned}
$$

\section{Propanoic acid}

$$
\begin{aligned}
\lambda= & \frac{\left[1.45 \times 10^{-7}+2.13 \times 10^{-7}\left(C_{p_{1}}\right)\right] T^{1.5}}{(T+429)} \\
& +\frac{\left(11.48+1.73 \times 10^{-3} T-1.98 \times 10^{-6} T^{2}\right)}{T^{0.5}(T+521)} \frac{\bar{x}_{1} x_{2}}{\left(2 x_{2}+x_{1}\right)^{2}}
\end{aligned}
$$

$\log P_{e}=7.9223-\frac{1869.4}{T-43.16}^{k}$

$\sigma=64.819-0.153 T+7.825 \times 10^{-5} T^{21}$

$\eta=114.5 \times 10^{-7} \frac{T^{1.5}}{(T+521)} \ominus$

$d=1.3087-0.0011 T^{1}$

$$
C_{p_{1}}=2.409+7.743 \times 10^{-2} T-4.606 \times 10^{-5} T^{2 \mathrm{~h}}
$$$$
C_{p_{2}}=4.818+1.549 \times 10^{-1} T-9.212 \times 10^{-5} T^{2 b}
$$

$$
\begin{aligned}
\log K_{12} & =\frac{2846.8}{T}-9.820^{\mathrm{m}} \\
M & =74.08 \\
D_{13} & =0.3015 \frac{T^{s}}{C} \\
s & =0.75^{j}
\end{aligned}
$$

Helium

$$
\begin{aligned}
\lambda & =7.376974 \times 10^{-5}+1.139222 \times 10^{-6} T-6.343536 \times 10^{-10} T^{2^{n}} \\
\eta & =145.5 \times 10^{-7}{\frac{T^{1.5}}{(T+74.1)}}^{\circ} \\
M & =4.0026
\end{aligned}
$$

${ }^{\lambda}$ in $\mathrm{cal} /(\mathrm{cm} \mathrm{sK}), P_{e}$ in $\mathrm{mm} \mathrm{Hg}, \sigma$ in $\mathrm{erg} / \mathrm{cm}^{2}, \eta$ in $\mathrm{P}, d$ in $\mathrm{g} /$

\section{TABLE I. (Continued)}

$\mathrm{cm}^{3}, C_{p}$ in cal/(mole K), $K_{12}$ in $\mathrm{mm}^{-1}, D_{13}$ in $\mathrm{cm}^{2} / \mathrm{s}, s$ is dimensionless, and $T$ in $\mathrm{K}$.

The first term is the thermal conductivity of pure monomer $\lambda_{f}$ and the second term is the thermal conductivity due to the chemical reaction $\lambda_{R} \quad \lambda_{f}$ was determined using the modified Eucken correction; high temperature viscosity data was estimated using the method described in J. O. Hirschfelder, C. F. Curtiss, and R. B. Bird, Molecular Thcory of Gases and Liquids (Wiley, New York, 1954), Chap. 8. $\lambda_{R}$ was determined using the method outlined by T. A. Renner, G. H. Kucera, and M. Blander, J. Chem. Phys. 66, 177 (1977).

'Obtained from data in (i) J.Timmermans, Physico-Chemical Constants of Pure Organic Compounds (Elsevier, New York, 1950), Vol. 1, p. 377; and (ii) the International Critical

Tables (McGraw-Hill, New York, 1928), Vol. 4, p. 448.

dobtained from data in J. Timmermans, footnote c(i), p. 379; and footnote c(ii), Vol. 4, p. 450 .

${ }^{e}$ Estimated using the method described in J. O. Hirschfelder, C. F. Curtiss, and R. B. Bird, Molecular Theory of Gases and Liquids (Wiley, New York, 1954), Chap. 8.

'Obtained from data in J. Timmermans, footnote c(i), p. 378; and J. B. Garner, B. Saxton, and H. O. Parker, Am. Chem. J. 46,239 (1911).

BObtained from the calculated values in J. H. S. Green, J. Chem. Soc. 1961, 2241.

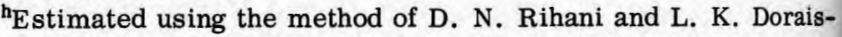
wamy, Ind. Eng. Chem. Fundam. 4, 17 (1965).

iJ. R. Barton and C. C. Hsu, J. Chem. Eng. Data 14, 184 (1969).

${ }^{\prime}$ E. N. Fuller, P. D. Schettler, and J. C. Giddings, Ind. Eng. Chem. 58, 19 (1966). The numerical coefficient represents the binary diffusion coefficient at $273.16 \mathrm{~K}$ and $1 \mathrm{~atm}$.

${ }^{k}$ R. R. Driesbach and S. A. Schrader, Ind. Eng. Chem. 41, 2879 (1949).

${ }^{1} \mathrm{~K}$. W. Hunten and O. Maass, J. Am. Chem. Soc. 51, 153 (1929).

m. Guilleme and B. Wojtkowiak, Bull. Soc. Chim. Fr. 41, 1282 (1974).

${ }^{n}$ Thermophysical Properties Research Center Data Book (Purdue University, West Lafayette, 1970), Vol. 3.

${ }^{\circ}$ Obtained from data in Onnes and Weber, Verh. K. Acad. Wet. Amsterdam Afd. Naturerkd. 21, 1385 (1913).

TABLE II. Experimental data for the formic acid and propanoic acid critical supersaturation measurements. $T_{0}$ is the surface temperature of the working fluid, $T_{1}$ is the surface temperature of the condensate film on the upper plate, and $P_{T}$ is the total pressure inside the cloud chamber.

\begin{tabular}{lclll}
\hline \hline & $\begin{array}{c}\text { Experiment } \\
\text { number }\end{array}$ & $T_{0}(\mathrm{~K})$ & $T_{1}(\mathrm{~K})$ & $P_{T}(\mathrm{~mm} \mathrm{Hg})$ \\
\hline Formic acid & 1 & 366.9 & 271.8 & 1240 \\
& 2 & 369.0 & 272.7 & 1163 \\
Propanoic acid & 1 & 311.0 & 237.6 & 77.0 \\
& 2 & 313.3 & 240.5 & 84.0 \\
& 3 & 315.1 & 242.8 & 97.0 \\
& 4 & 317.7 & 245.2 & 117.0 \\
& 5 & 319.8 & 249.1 & 95.0 \\
& 6 & 324.4 & 252.7 & 159.0 \\
& 7 & 327.4 & 257.2 & 118.0 \\
& 8 & 330.4 & 261.3 & 111.0 \\
& 9 & 345.2 & 271.7 & 137.0 \\
& 10 & 349.1 & 277.5 & 165.8 \\
& 11 & 349.3 & 279.7 & 563.0 \\
& 12 & 354.2 & 286.7 & 736.5 \\
\hline
\end{tabular}




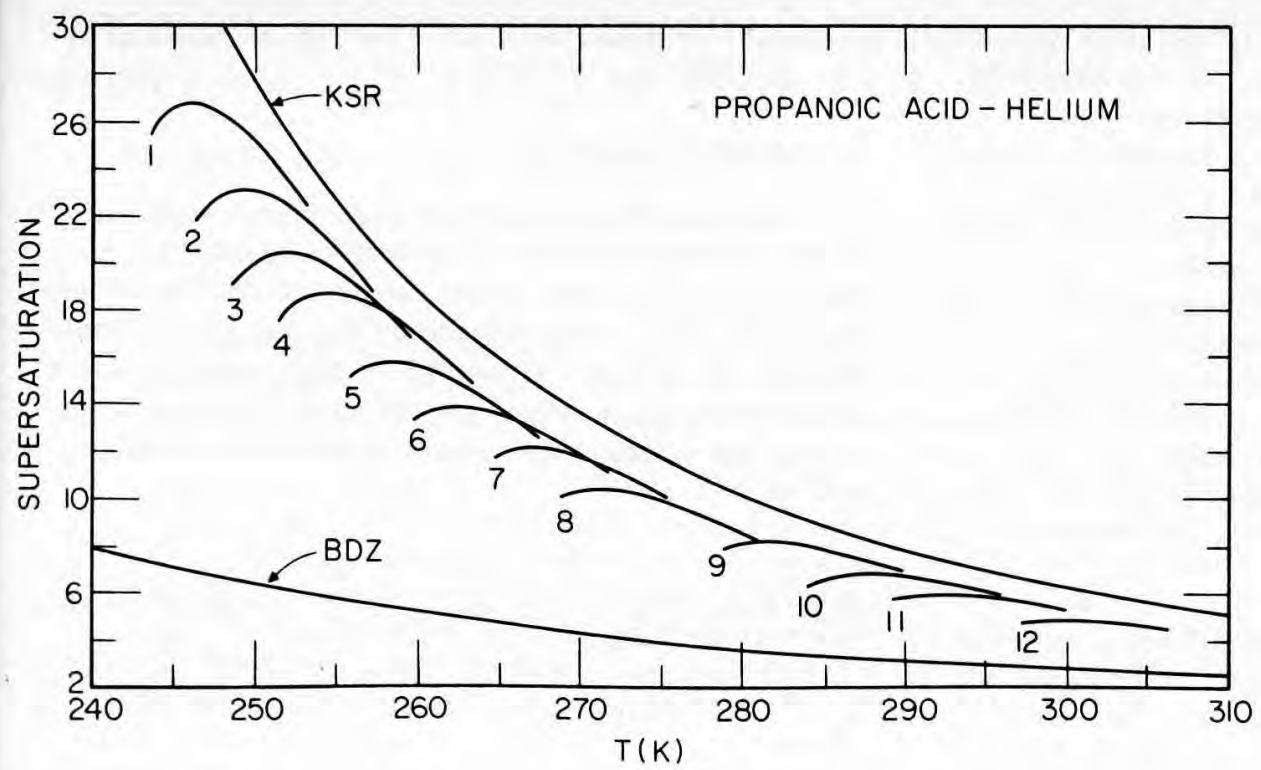

FIG. 2. The variation of the critical supersaturation of propanoic acid vapor as a function of temperature. The curves labeled BDZ and KSR are the predictions of the Becker-Döring-Zeldovitch and Katz-Saltsburg-Reiss theories, respectively. The envelope of the numbered curves is the experimental result.

would have to be increased by roughly $10^{20}$ for formic acid and $10^{16}$ for propanoic acid to have the experimental critical supersaturation agree with the BDZ prediction. This is the same sort of behavior observed for acetic acid. ${ }^{5}$

The solid curve in Figs. 1 and 2 labeled KSR is the variation of critical supersaturation with temperature given by the Katz-Saltsburg-Reiss theory of nucleation in associated vapors. ${ }^{1}$ The role of association in nucleation appears to be twofold: a kinetic effect whereby the growth of a precritical embryo is implemented in an $m$-sized jump by acquisition of an association cluster containing $m$ monomers; and a thermodynamic effect in which the embryo distribution is skewed by the presence of the association clusters, the former tending to in crease the nucleation rate while the latter decreases it. The thermodynamic effect is generally the more important of the two, and the net nucleation rate is decreased.

The rate of nucleation in the Katz-Saltsburg-Reiss model in drops $/\left(\mathrm{cm}^{3} \mathrm{~s}\right)$ is given by

$$
\begin{aligned}
J= & \frac{a}{d}\left(\frac{2 N_{0}^{3} \sigma M}{\pi}\right)^{1 / 2}\left(\frac{S P_{e}}{R T}\right)^{2} \sum_{i=1}^{m} i^{3 / 2} x_{i} \\
& \times \exp \left[-\frac{16 \pi N_{0}}{3\left(\ln S_{1}\right)^{2}}\left(\frac{M}{d}\right)^{2}\left(\frac{\sigma}{R T}\right)^{3}\right] .
\end{aligned}
$$

In Eq. (6), the upper summation index $m$ is the number of monomers in the largest association cluster, $x_{i}$ is $P_{i} / P$, where $P_{i}$ is the partial pressure of the association clusters of size $i, P$ is the existing pressure of the vapor, and $S_{1}$ is the monomer supersaturation ratio $P_{1} / P_{1 e}$, where $P_{1 e}$ is the equilibrium vapor pressure of the monomer. The curves labeled KSR in Figs, 1 and 2 were obtained by setting $J=1 \mathrm{drop} /\left(\mathrm{cm}^{3} \mathrm{~s}\right)$ in Eq. (6), assuming a value of unity for the accommodation coefficient and solving for $S$, the total supersaturation, at a particular temperature. Values for the liquid density, surface tension, equilibrium vapor pressure, and the equilibrium monomer-dimer association constant used in Eqs. (5) and (6) are given in Table I.
For both formic and propanoic acids, the KSR theory appears to give the correct temperature dependence of the critical supersaturation. The actual value of the critical supersaturation at any particular temperature for formic acid appears to be in agreement with the theory, while it is roughly $14 \%$ lower in the case of propanoic acid. There is some question regarding the formic acid data, however, largely because we were restricted to a rather narrow range of operating temperatures. Apart from the difficulties of working with formic acid described earlier, the large difference in surface temperatures necessary for nucleation $\left(T_{0}-T_{1}\right.$ $\sim 95 \mathrm{~K})$ as opposed to the relatively small difference between the boiling and melting points $(\sim 92 \mathrm{~K})$ seriously limited the temperature range available for measurement. Why this creates a problem is related to the (observed) fact that, in order to obtain reliable, reproducible experimental data with the diffusion cloud chamber, the ratio of the total pressure to the equilibrium vapor pressure at the lower surface temperature must, in general, be greater than some minimum value $p_{r}$. While this idea has been discussed in detail in the past, ${ }^{7,24}$ the important point is that this minimum value must be determined for each different working fluid. For instance, the value of $p_{r}$ for propanoic acid was found to be approximately 3 . In all our measurements on formic acid, the largest pressure ratio we achieved was slightly greater than 2 , the reason being that the temperature of the liquid pool is always near its boiling point so the total pressure must be rather high; and, as we are using a Pyrex ring to separate the chamber plates, we are necessarily limited to moderate total pressures ( $\$ 1300 \mathrm{~mm} \mathrm{Hg}$ ) for fear of rupturing the ring. The reason we were able to make the nucleation measurements at all is due to the fact that formic acid, as well as propanoic acid, undergoes a rather large degree of supercooling. Ten degrees of supercooling for formic acid on the upper plate surface was common; while propanoic acid could be supercooled by as much as $15 \mathrm{~K}$. The degree of supercool was not as crucial for propanoic acid as it was for formic, but it did extend the accessible temperature range. 
Homogeneous nucleation measurements were made with both the $99.2 \%$ pure formic acid and the $97.6 \%$ as is material obtained from the manufacturer. The results in both cases were not significantly greater than our experimental error. It should be remembered, however, that in both cases there is the inherent uncertainty due to the low pressure ratio. Also, regarding the question of impurities, there is the possibility of an impurity being produced in situ because of the acid attack upon the gasket material. While we cannot rule this out completely with the formic acid experiments, critical supersaturation data for propanoic acid are independent of gasket history (old, new, etc.) and the length of time the working fluid was in the chamber (several hours or several days).

Formic acid undergoes a continuous thermal decomposition to water and carbon monoxide. During our experiments, the pool of formic acid was maintained at temperatures in excess of $365 \mathrm{~K}$ for several hours so it was necessary to determine the extent of the decomposition and the effect, if any, on the supersaturation. Using literature values for the rate of thermal decomposition of formic acid, ${ }^{8}$ the composition of the pool was found to change by less than $1.2 \%$ during any one experiment. This change in composition changes the vapor pressure of formic acid by roughly $2 \%,{ }^{25}$ and this decreases the critical supersaturation by only $2 \%$. Since fresh formic acid was used for every experiment, and a $2 \%$ variation in critical supersaturation was less than the experimental uncertainty, the effect of the thermal decomposition was ignored.

During our experiments, we applied electric fields of up to $140 \mathrm{~V} / \mathrm{cm}$ across the chamber plates to sweep out any natural occurring ions in order to avoid confusing ion-induced nucleation with actual homogeneous nucleation. In all cases, we could discern no change in nucleation rate with or without the electric field for either formic or propanoic acids. This same behavior was also observed with acetic acid. ${ }^{5}$ It seems that, at least for the naturally occurring background concentration of charged particles, the onset of ion-induced nucleation is always preceded by homogeneous nucleation for formic, acetic, and propanoic acids. ${ }^{26}$ Interestingly, this is just the opposite sort of behavior one observes in the case of water 6 and the lower molecular weight alcohols. ${ }^{27,28}$

The extent to which the solutions of Eqs. (2) and (3) depend upon the values of the various parameters listed in Table I has been discussed in I and will not be reproduced in detail here. It should be mentioned, however, that the effect the uncertainty in the thermal conductivity, binary diffusion coefficient, heat capacity, and vapor viscosity discussed earlier in this paper has upon the pressure and temperature profiles and ultimately the supersaturation is relatively small. For instance, variations of $10 \%$ in the binary diffusion coefficient or the vapor viscostiy change the calculated supersaturation by less than $2 \%$. A $50 \%$ change in the acid thermal conductivity produces only a $1 \%$ change in the supersaturation and finally a $10 \%$ variation in heat capacity (monomer or dimer) will alter the supersaturation by less than $2 \%$

\section{ACKNOWLEDGMENTS}

This research was partially supported by the National Science Foundation under Grant \#ENG75-15342. Acknowledgment is made to the Donors of The Petroleum Research Fund, administered by the American Chemical Society, for the partial support of this research under Grant \#8296-G6, 7. One of us (Y.G.R.) acknowledges support as a Dupont Fellow during a portion of this research.

${ }^{1}$ J. L. Katz, H. Saltsburg, and H. Reiss, J. Colloid Interface Sci. 21, 560 (1966).

${ }^{2}$ H. L. Frisch and C. Willis, J. Chem. Phys. 22, 243 (1954).

${ }^{3}$ T. H. Laby, Philos. Trans. R. Soc. London Ser. A 208, 445 (1908).

${ }^{4}$ For instance, Laby neglected the presence of the organic vapor in determining the ratio of the heat capacities. This has more than the obvious consequence since the enthalpy change accompanying the association reaction can have a pronounced effect upon the heat capacity.

${ }^{5}$ R. H. Heist, K. M. Colling, and C. S. Dupuis, J. Chem. Phys. 65, 5147 (1976).

${ }^{6}$ R. H. Heist, and H. Reiss, J. Chem. Phys. 59, 665 (1973).

${ }^{7}$ J. L. Katz, J. Chem. Phys. 65, 382 (1976).

${ }^{8}$ H. N. Barham and L. W. Clark, J. Am. Chem. Soc. 73, 4638 (1951).

9 J. R. Barton and C. C. Hsu, J. Chem. Eng. Data 14, 184 (1969).

${ }^{10} \mathrm{~J}$. Guillme and B. Wojtkowiak, Bull. Soc. Chim. Fr. 41, 1282 (1974).

${ }^{11}$ M. D. Taylor and J. Bruton, J. Am. Chem. Soc. 74, 4151 (1952).

${ }^{12}$ C. Becker, H. Reiss, and R. H. Heist, J. Chem. Phys. 68, 3585 (1978)

${ }^{13}$ W. Nernst, Boltzmann-Festschr. 904 (1904).

${ }^{14}$ A. A. Tarzimanov and V. E. Mashirov, Thermophysical Properties of Matter and Substances, edited by V. A. Rabinovich (Amerind, New Delhi, 1974), Vol. 2, Chap. 3.

${ }^{15} \mathrm{~J}$. N. Butler and R. S. Brokaw, J. Chem. Phys. 26, 1636 (1957).

${ }^{16}$ R. S. Brokaw, J. Chem. Phys. 35, 1569 (1961).

${ }^{17}$ T. A. Renner, G. H. Kucera, and M. Blander, J. Chem. Phys. 66, 177 (1977).

${ }^{18} \mathrm{~J}$. O. Hirschfelder, C. F. Curtiss, and R. B. Bird, Molecular Theory of Gases and Liquids (Wiley, New York, 1954), Chap. 8.

${ }^{19}$ J. H. S. Green, J. Chem. Soc. 1961, 2241.

${ }^{20}$ D. N. Rihani and L. K. Doraiswamy, Ind. Eng. Chem. Fundam. 4, 17 (1965).

${ }^{21}$ W. Weltner, J. Am. Chem. Soc. 77, 3941 (1955).

${ }^{2 i}$ D. N. Seshadri, O. S. Viswanath, and N. R. Kuloon, A.I. Ch. E. J. 16, 420 (1970).

${ }^{23}$ E. N. Fuller, P. D. Schettler, and J. C. Giddings, Ind. Eng. Chem. 58, 19 (1966).

${ }^{24}$ K. M. Colling, M. S. Thesis, University of Rochester (1976).

${ }^{25}$ F. Rivenq, Bull. Soc. Chim. Fr. 27, 1505 (1960).

${ }^{26}$ In Ref. 3, Laby observed that irradiating his cloud chamber with $\mathrm{x}$ rays did produce ion-induced nucleation at lower supersaturations than required for homogeneous nucleation.

${ }^{27}$ J. L. Katz and B. J. Ostermier, J. Chem. Phys. 47, $478^{\circ}$ (1967).

${ }^{28}$ C. S. Dupuis, M. S. Thesis, University of Rochester (1978). 